\title{
Asymmetric dimethylarginine concentrations are elevated in women with gestational diabetes
}

\author{
Mujde Akturk • Alev Altinova $\cdot$ Ismail Mert • \\ Aylin Dincel · Ayla Sargin · Umran Buyukkagnici • \\ Metin Arslan · Nuri Danisman
}

Received: 9 September 2009/Accepted: 19 April 2010/Published online: 11 July 2010

(C) Springer Science+Business Media, LLC 2010

\begin{abstract}
As shown in the previous studies, asymmetric dimethylarginine (ADMA) is related to endothelial dysfunction, whereas high-sensitive C-reactive protein (hCRP) is the marker of inflammation. In our study, we investigated ADMA, hCRP, and homocysteine concentrations in women with gestational diabetes mellitus (GDM) and normal glucose tolerance (NGT) during late pregnancy. Fifty-four women with GDM and 69 women with NGT between 32 and 39 weeks of gestation were included in this study. ADMA, hCRP, homocysteine, lipid parameters, glycated hemoglobin (HbA1c) levels, insulin, and homeostasis model assessment for insulin resistance (HOMA-IR) were measured. The plasma ADMA concentrations were
\end{abstract}

M. Akturk · M. Arslan

Department of Endocrinology and Metabolism, Faculty

of Medicine, Gazi University, Ankara, Turkey

\section{A. Altinova}

Division of Endocrinology, Zekai Tahir Burak Women's Health Education and Research Hospital, Ankara, Turkey

I. Mert · A. Sargin · N. Danisman

Department of Perinatology, Zekai Tahir Burak Women's Health

Education and Research Hospital, Ankara, Turkey

\author{
A. Dincel \\ Department of Biochemistry, Faculty of Medicine, Gazi \\ University, Ankara, Turkey \\ U. Buyukkagnici \\ Division of Biochemistry, Zekai Tahir Burak Women's Health \\ Education and Research Hospital, Ankara, Turkey \\ M. Akturk ( $\square)$ \\ Bilkent University, Merkez Loj. 21/5, Bilkent, 06800 Ankara, \\ Turkey \\ e-mail: mujdeakturk@hotmail.com
}

significantly higher in GDM patients than in NGT subjects $(P=0.03)$ and the hCRP levels were also significantly increased in GDM group when compared with those in the NGT group $(P=0.008)$. However, plasma homocysteine levels did not differ between the groups $(P=0.4)$, while HOMA-IR, insulin, and triglyceride levels were higher in the GDM group than in the NGT group $(P=0.001,0.002$, and 0.02 , respectively). The ADMA concentrations in the third trimester were positively correlated with the glucose levels the 50-g glucose challenge test (GCT) during 24-28 weeks in the whole group $(r=0.21, P=0.02)$. Our results demonstrate that ADMA and hCRP are elevated in women with GDM during late pregnancy. Further studies are needed to clarify the significance and the underlying mechanisms of the elevated ADMA and hCRP levels in women with GDM.

Keywords Asymmetric dimethylarginine . Gestational diabetes $\cdot$ High-sensitive C-reactive protein (hCRP) $\cdot$ Homocysteine $\cdot$ Insulin resistance

\section{Introduction}

Pregnancy is a state of insulin resistance, which emerges in the second trimester and is most prominent in the late third trimester. In some predisposed women, whose pancreas' functions cannot overcome insulin resistance, gestational diabetes mellitus (GDM) is manifested [1]. GDM is defined as the abnormal glucose metabolism, first diagnosed during pregnancy, and is one of the most common complications of pregnancy $[1,2]$. Subjects who have a history of GDM are shown to have endothelial dysfunction [3, 4], elevated cardiovascular risk [1], and are also at increased risk of 
developing impaired glucose tolerance or type-2 diabetes [1]. Moreover, recent evidence suggests that cardiovascular risk is increased in previous GDM even if they do not have metabolic abnormality [5, 6].

Atherosclerosis is one of the most common causes of mortality and morbidity. Pathogenesis of atherosclerosis includes endothelial dysfunction, inflammation, and elevated levels of homocysteine and lipids [7]. Nitric oxide (NO) is an endothelium-derived vasoactive mediator, which is produced by NO synthase from L-arginine. As such, NO is considered to be an anti-atherosclerotic molecule [8]. Asymmetric dimethylarginine (ADMA) has been found to be an endogenous inhibitor of NO synthase [9]. ADMA can cause endothelial dysfunction by inhibiting NO synthesis [10], and it is known that this inhibition underlies one of the main pathogenesis of cardiovascular disease [11, 12] and is associated with type- 1 diabetes before the development of vascular complications [13]. Additionally, high-sensitive $\mathrm{C}$-reactive protein (hCRP) and homocysteine levels are also considered to be atherosclerosis-related markers. Homocysteine is an amino acid synthesized by demethylation of methionine, and increased homocysteine levels are found to be associated with atherosclerosis [14]. hCRP is an important marker of inflammation and the results of a recent study suggest that inflammation may also be an underlying pathophysiological mechanism of GDM, which is associated with insulin resistance [15]. ADMA and hCRP were found to be the best predictors of the increased intima media thickness in patients with chronic renal failure [16]. In type-2 diabetic patients, ADMA both predicted and elevated the predictive value of CRP in cardiovascular disease [17].

The ADMA levels measured after delivery were shown to be increased in women with previous GDM, when compared with the control subjects [18]. In a follow-up study of GDM patients, elevated ADMA concentrations after delivery were found to be associated with deterioration in glucose tolerance in women with previous GDM [19]. To our knowledge, the study by Telejko et al. [20] is the only published study that investigated the ADMA levels in GDM patients during pregnancy and compared the ADMA levels among second trimester GDM, normal glucose tolerance (NGT), and non-pregnant healthy control subjects. They found that the ADMA levels were not significantly different between GDM and NGT groups; however, both the groups had lower ADMA levels than nonpregnant healthy subjects, and the authors explained this difference as a normal adaptation to pregnancy [20].

In this study, our aim is to investigate the circulating ADMA levels and other atherosclerosis markers involving hCRP, homocysteine levels, and lipid parameters in women with GDM when compared with women with NGT during late pregnancy.

\section{Patients and methods}

Subjects and study design

A total of 123 pregnant women from Obstetrics and Gynecology and Endocrinology Departments, comprising 54 GDM women with a mean age of 29.70 years (age range: $17-40$ years) and 69 NGT women with a mean age of 28.60 years (age range: 18-40 years), between 32 and 39 weeks of gestation were included in this study. All obstetrical and personal information were obtained using a questionnaire. The mean gestational weeks for GDM and NGT women were $35.53 \pm 0.23$ and $36.37 \pm 0.22$ weeks, respectively. The mean body mass index (BMI) of the GDM group was $29.59 \pm 0.58 \mathrm{~kg} / \mathrm{m}^{2}$ and that of NGT group was $28.66 \pm 0.47 \mathrm{~kg} / \mathrm{m}^{2}(P>0.05)$. A total of 115 pregnant women underwent $50-\mathrm{g}$ glucose challenge test (GCT) between 24 and 28 weeks. Oral glucose tolerance test (OGTT) with 100-g glucose was performed in subjects with 1 -h glucose level $>140 \mathrm{mg} / \mathrm{dl}$ in GCT. GDM was defined if two or more abnormal values were observed in OGTT (normal glucose values are: fasting $<95,1$ - $\mathrm{h}<180$, 2-h $<155$, and 3-h $<140 \mathrm{mg} / \mathrm{dl}$ ) according to the diagnostic criteria of the American Diabetes Association [1]. All the subjects in the control group were rechecked using fasting blood glucose (FBG) and 1-h post-prandial glucose (PPG) level in the late third trimester, and their FBG and PPG levels were $74.05 \pm 1.11$ and $105.4 \pm 2.8 \mathrm{mg} / \mathrm{dl}$, respectively. The exclusion criteria were defined as any systemic disease known to affect ADMA and inflammatory markers, recent infection, chronic hypertension, gestational-induced hypertension, preeclampsia and eclampsia, pregnancies of assisted reproductive techniques, recent betamethosone use, and multiple pregnancies. None of the pregnant women were in active labor. A total of 48 GDM women were treated using diet only, while 6 women were treated using both insulin and diet. This project was approved by Local Ethical Review Board of Zekai Tahir Burak Women's Health Hospital, and informed consent was taken from all the participants.

\section{Sample analyses}

Laboratory tests included determining the levels of ADMA, hCRP, homocysteine, total cholesterol, low-density lipoprotein (LDL), high-density lipoprotein (HDL), triglycerides (TG), serum fasting glucose, insulin, and glycated hemoglobin (HbAlc) (only for diabetic patients). PPG were also performed $1 \mathrm{~h}$ after the meal. The blood samples were collected after an overnight fasting, and were immediately separated and stored at $-80^{\circ} \mathrm{C}$ until for ADMA analysis. The plasma ADMA levels were measured by high performance liquid chromatography (HPLC)- 
fluorescence detector using the method described by Chen et al. [21], while serum hCRP was measured using highsensitive immunoturbidimetric assay (Roche Modular P automated clinical chemistry analyzer, Roche Diagnostics GmBH, Mannheim, Germany). Furthermore, plasma homocysteine levels were measured by competitive immunoassay method using the Immulite homocysteine kit (Siemens, Los Angeles, California) on an Immulite analyzer, and $\mathrm{HbAlc}$ levels were measured using turbidimetric inhibition assay (Roche Diagnostics GmBH, Mannheim, Germany). Serum glucose levels were measured with standard enzymatic methods (Roche Diagnostics GmBH, Mannheim, Germany), and serum concentrations of total cholesterol, HDL-cholesterol, and TG were measured by enzymatic calorimetric method using commercially available kits (Roche Diagnostics GmBH, Mannheim, Germany). LDL-cholesterol levels were calculated using the Friedewald equation, and insulin levels were measured by immunoradiometric assay (sandwich-type assay) using an insulin IRMA kit (Immunotech, Prague, Czech Republic). Furthermore, insulin sensitivity was determined using the homeostasis model assessment for insulin resistance (HOMA-IR), calculated as [serum glucose level $(\mathrm{mmol} / \mathrm{l}) \times$ insulin $\mu \mathrm{IU} / \mathrm{ml}) / 22.5]$. For each pregnant woman, the BMI was calculated as weight in kilograms divided by height in meters squared. Folic acid levels were measured by competitive immunoassay method and Vitamin B12 levels were measured using solid phase, competitive chemoluminescent enzyme immunoassay method (Immulite Folic acid-Siemens and Immulite VitB12-Siemens).

\section{Statistical analysis}

In this study, the statistical analysis was performed using the SPSS version 15.0. The data were expressed as mean \pm standard error of mean (SEM), unless specified otherwise. To determine whether differences existed between the groups, Mann-Whitney $U$ test was employed, and $P$ values below 0.05 were considered to be statistically significant. For univariate correlation between continuous variables, Spearman coefficients were used. Correlation analysis between the parameters was carried out for the whole group and GDM and NGT groups separately. Multivariate analysis was performed using Enter regression analysis, and before the analysis, the non-normally distributed data were logarithmically transformed.

\section{Results}

Some demographic and biochemical characteristics of the pregnant women with GDM and NGT are presented in
Table 1. The plasma ADMA concentrations were significantly higher in GDM patients than in NGT patients (1.37 \pm 0.11 (median 1.1 ) vs. $1.08 \pm 0.07$ (median 0.89 ) $\mu \mathrm{mol} / \mathrm{l}$, $P=0.03$ ) (see Fig. 1). The serum hCRP levels were also significantly elevated in GDM patients $(9.43 \pm 1.13$ (median 6.8), $6.31 \pm 0.69$ (median 4.8 ) $\mathrm{mg} / \mathrm{l}$, respectively, $P=$ 0.008). However, plasma homocysteine concentrations did not differ between the groups $(5.20 \pm 0.30$ (median 4.9) vs. $5.62 \pm 0.34$ (median 5.2) $\mu \mathrm{mol} / \mathrm{l}, P=0.4$ ). Furthermore, HOMA-IR and insulin levels were significantly higher in GDM group than in NGT group $(5.23 \pm 0.91$ vs. $3.13 \pm$ $0.45, P=0.001$ and $24.40 \pm 3.27$ vs. $16.80 \pm 2.25 \mu \mathrm{IU} / \mathrm{ml}$, $P=0.002$, respectively). The TG levels were also significantly elevated in the GDM group $(272.98 \pm 17.76$ vs. $231.00 \pm 9.84 \mathrm{mg} / \mathrm{dl}, P=0.02$ ), while the ADMA levels were not different between the GDM patients who were treated with diet and diet plus insulin $(1.37 \pm 0.12$ vs. $1.35 \pm 0.36 \mu \mathrm{mol} / \mathrm{l}, P=0.6$ ).

The Spearman correlation analyses of ADMA, hCRP, homocysteine, and the selected variables are summarized in Tables 2 and 3. As shown in Fig. 2, the ADMA levels in the third trimester were positively correlated with 1-h glucose at 50-g GCT levels during 24-28 weeks in the whole group $(r=0.21, P=0.02)$. Spearman correlation did not show any correlation between ADMA levels and age, gestational week, BMI, prepregnancy BMI, FBG, PPG, hCRP, HbA1c, insulin, HOMA-IR, TC, TG, HDLcholesterol, and LDL-cholesterol levels of the GDM group and control group $(P>0.05)$. In the NGT group, the ADMA levels were positively correlated with the homocysteine levels $(r=0.28, P=0.03)$.

Homocysteine levels were positively correlated with age $(r=0.20, P=0.03)$ and TC $(r=0.21, P=0.02)$, and negatively correlated with folic acid levels $(r=-0.36$, $P<0.001)$ in the whole group. Furthermore, the homocysteine levels were negatively correlated with folic acid $(r=-0.61, P<0.001)$, positively correlated with 1-h glucose at 50-g GCT $(r=0.27, P=0.04)$ and ADMA concentrations $(r=0.28, P=0.03)$ in the NGT group, and positively correlated with TG $(r=0.31, P=0.02)$ in the GDM group. By using multiple regression analysis with log homocysteine as the dependent variable and age, folic acid, log TG, log ADMA, and log 1-h glucose at 50-g GCT, we found negative relationship between log homocysteine and folic acid levels $(B=-0.29, P=0.004)$ and $\log$ TG levels $(B=0.21, \quad P=0.03) \quad\left(r^{2}=0.15\right.$ and $R=0.39$ ).

The hCRP levels were correlated with BMI in both the GDM $(r=0.30, P=0.03)$ and NGT groups $(r=0.30$, $P=0.01)$ as well as the whole group $(r=0.32$, $P<0.001)$. Furthermore, there was a positive correlation between the hCRP levels and prepregnancy BMI in the whole group $(r=0.19, P=0.04)$. In the GDM group, the 
Table 1 Clinical characteristics and biochemical results of GDM and NGT groups

\begin{tabular}{|c|c|c|c|}
\hline Parameter & GDM & NGT & $P$ value \\
\hline$n$ & 54 & 69 & \\
\hline Age (year) & $29.70 \pm 0.67$ & $28.60 \pm 0.64$ & 0.3 \\
\hline $\operatorname{BMI}\left(\mathrm{kg} / \mathrm{m}^{2}\right)$ & $29.59 \pm 0.58$ & $28.66 \pm 0.47$ & 0.1 \\
\hline Prepregnancy BMI $\left(\mathrm{kg} / \mathrm{m}^{2}\right)$ & $25.53 \pm 0.64$ & $23.69 \pm 0.49$ & $0.02 *$ \\
\hline $50-\mathrm{g} \mathrm{GCT}(\mathrm{mg} / \mathrm{dl})(n=115)$ & $188.75 \pm 6.9$ & $100.75 \pm 2.46$ & $<0.001 * *$ \\
\hline FBG (mg/dl) & $81.40 \pm 2.65$ & $74.05 \pm 1.11$ & 0.07 \\
\hline PPG (mg/dl) & $127.17 \pm 4.26$ & $105.4 \pm 2.80$ & $<0.001 * *$ \\
\hline Insulin $(\mu \mathrm{IU} / \mathrm{ml})$ & $24.40 \pm 3.27$ & $16.80 \pm 2.25$ & $0.002 * *$ \\
\hline HOMA-IR & $5.23 \pm 0.91$ & $3.13 \pm 0.45$ & $0.001 * *$ \\
\hline $\mathrm{HbA1c}(\%)$ & $5.46 \pm 0.09$ & - & \\
\hline $\mathrm{TC}(\mathrm{mg} / \mathrm{dl})$ & $246.75 \pm 4.51$ & $250.10 \pm 5.82$ & 0.9 \\
\hline HDL-C (mg/dl) & $73.20 \pm 2.31$ & $77.82 \pm 2.24$ & 0.1 \\
\hline LDL-C (mg/dl) & $117.71 \pm 5.93$ & $126.60 \pm 5.08$ & 0.2 \\
\hline TG $(\mathrm{mg} / \mathrm{dl})$ & $272.98 \pm 17.76$ & $231.00 \pm 9.84$ & $0.02 *$ \\
\hline ADMA $(\mu \mathrm{mol} / 1)$ & $1.37 \pm 0.11$ & $1.08 \pm 0.07$ & $0.03 *$ \\
\hline hCRP (mg/l) & $9.43 \pm 1.13$ & $6.31 \pm 0.69$ & $0.008 * *$ \\
\hline Homocysteine $(\mu \mathrm{mol} / \mathrm{l})$ & $5.20 \pm 0.30$ & $5.62 \pm 0.34$ & 0.4 \\
\hline Folic acid (ng/ml) & $12.99 \pm 0.75$ & $11.14 \pm 0.68$ & 0.07 \\
\hline Vitamin B12 (pg/ml) & $208.78 \pm 10.43$ & $204.14 \pm 10.91$ & 0.7 \\
\hline
\end{tabular}

Data were expressed as mean \pm standard error of mean (SEM)

GDM gestational diabetes mellitus, $N G T$ normal glucose tolerance, ADMA asymmetric dimethylarginine, $h C R P$ high-sensitive C-reactive protein, $B M I$ body mass index, HOMA-IR homeostasis model assessment for insulin resistance, $F B G$ fasting blood glucose, $P P G$ post-prandial blood glucose, $50 \mathrm{~g} G C T$ blood glucose levels after $1 \mathrm{~h} 50$-g glucose challenge test, $T C$ total cholesterol, $L D L$ - $C$ low-density lipoprotein cholesterol, $H D L-C$ high-density lipoprotein cholesterol, $T G$ triglycerides

$P>0.05$ statistically non-significant, $* P<0.05$, ** $P<0.01$

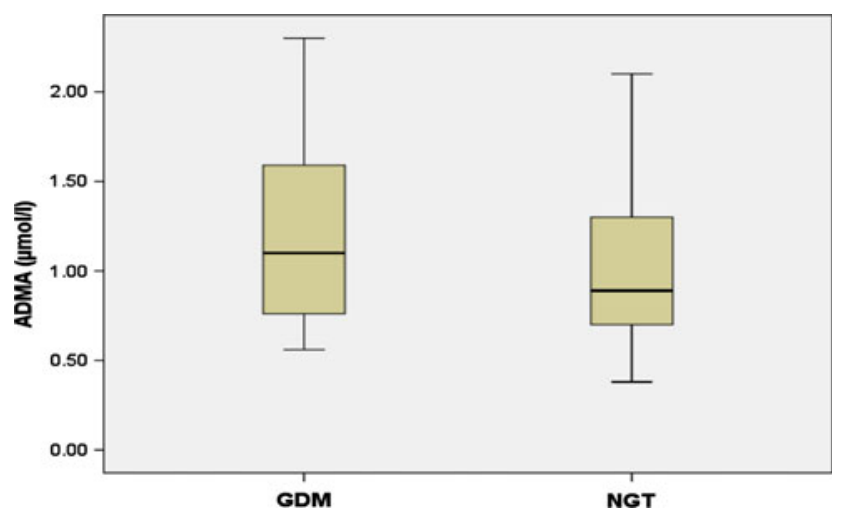

Fig. 1 ADMA levels are higher in GDM group than in the NGT group

hCRP levels were positively correlated with HbA1c $(r=0.46, P<0.001)$.

\section{Discussion}

Our study showed that the plasma ADMA concentration were higher in women with GDM than NGT during pregnancy between 32 and 39 weeks of gestation. In a recent study, the second trimester ADMA levels were compared between GDM and NGT subjects, where GDM patients had slightly higher ADMA concentrations than NGT subjects; however, a significant difference could not be determined [20]. As our study was performed during late third trimester, which is a relatively advanced and hence a more insulinresistant period of gestation, this might be the underlying mechanism responsible for higher ADMA levels in the GDM group. In addition, Mittermayer et al. [18] also showed that previous GDM women had increased ADMA levels even at a short time, such as 14-16 weeks, after delivery than NGT women. However, after a median of 2.75 years of follow-up, they found decreased ADMA concentrations irrespective of the changes in glucose tolerance [19]. ADMA, which is related with endothelial dysfunction, has been suggested as a marker of cardiovascular disease [8, 12]. Knock et al. [22] demonstrated the endothelial dysfunction in the subcutaneous arteries of GDM patients. Women with GDM may manifest short-term endothelial dysfunction during late pregnancy, which is manifested as transient hypertension [1]. As our study did not include any hypertensive subjects, increased ADMA concentration in late third trimester with 
Table 2 Spearman correlation analyses between ADMA, hCRP, homocysteine, and the selected variables in the whole group

\begin{tabular}{|c|c|c|c|c|c|c|}
\hline & \multicolumn{6}{|c|}{ Whole group } \\
\hline & \multicolumn{2}{|l|}{ ADMA } & \multicolumn{2}{|c|}{ Homocysteine } & \multicolumn{2}{|l|}{ hCRP } \\
\hline & $r$ & $P$ & $r$ & $P$ & $r$ & $P$ \\
\hline ADMA & - & & 0.10 & 0.2 & 0.10 & 0.2 \\
\hline Homocysteine & 0.10 & 0.2 & - & & 0.04 & 0.6 \\
\hline hCRP & 0.10 & 0.2 & 0.04 & 0.6 & - & \\
\hline Age & 0.08 & 0.3 & 0.20 & $0.03 *$ & -0.02 & 0.7 \\
\hline $\begin{array}{l}\text { Gestational } \\
\text { week }\end{array}$ & -0.06 & 0.4 & 0.07 & 0.4 & -0.02 & 0.8 \\
\hline BMI & 0.03 & 0.6 & -0.09 & 0.3 & 0.32 & $<0.001 * *$ \\
\hline $\begin{array}{l}\text { Prepregnancy } \\
\text { BMI }\end{array}$ & 0.12 & 0.1 & -0.05 & 0.5 & 0.19 & $0.04 *$ \\
\hline 50-g GCT & 0.21 & $0.02 *$ & 0.03 & 0.7 & 0.14 & 0.1 \\
\hline FBG & 0.06 & 0.4 & -0.04 & 0.6 & -0.10 & 0.2 \\
\hline HOMA-IR & 0.08 & 0.3 & -0.18 & 0.06 & 0.08 & 0.3 \\
\hline Insulin & 0.06 & 0.4 & -0.18 & 0.06 & 0.10 & 0.2 \\
\hline PPG & 0.01 & 0.9 & 0.01 & 0.9 & 0.07 & 0.4 \\
\hline $\mathrm{TC}$ & 0.01 & 0.9 & 0.21 & $0.02 *$ & 0.02 & 0.7 \\
\hline LDL-C & -0.01 & 0.1 & 0.10 & 0.2 & -0.02 & 0.8 \\
\hline TG & 0.07 & 0.3 & 0.15 & 0.1 & -0.008 & 0.9 \\
\hline HDL-C & 0.16 & 0.07 & 0.06 & 0.5 & 0.05 & 0.5 \\
\hline Folic acid & -0.04 & 0.6 & -0.36 & $<0.001 * *$ & -0.08 & 0.3 \\
\hline B12 & -0.005 & 0.9 & -0.10 & 0.2 & 0.04 & 0.6 \\
\hline
\end{tabular}

ADMA asymmetric dimethylarginine, $h C R P$ high-sensitive $\mathrm{C}$-reactive protein, $B M I$ body mass index, HOMA-IR homeostasis model assessment for insulin resistance, $F B G$ fasting blood glucose, $P P G$ post-prandial blood glucose, 50-g GCT blood glucose levels after $1 \mathrm{~h}$ $50-\mathrm{g}$ glucose challenge test, $T C$ total cholesterol, $L D L-C$ low-density lipoprotein cholesterol, $H D L-C$ high-density lipoprotein cholesterol, $T G$ triglycerides

$P>0.05$ statistically non-significant, $* P<0.05,{ }^{*} * P<0.01$

Bold values indicate statistically significant relationships

GDM patients might be related with endothelial dysfunction, even if they are not hypertensive.

Although ADMA levels did not correlate with FBG and PPG in late third trimester, we found a positive correlation between ADMA levels in the late third trimester and 1-h 50-g GCT values during 24-28 weeks. This positive correlation was statistically significant even though it was not too strong. However, we are unable to determine the exact pathological mechanisms that cause this relationship. Nevertheless, it is presumed that high glucose concentrations might increase the ADMA concentration by impairing the activity of the ADMA degrading enzyme, dimethylarginine dimethylaminohydroxylase (DDAH) [23]. DDAH activity can also be reduced in vitro by oxidative stress [23]. GDM has been linked to increase in free-radical formation [24], which might increase the ADMA level by inhibiting the DDAH activity [23].
We investigated the relationship between ADMA levels and HOMA-IR, because the relationship between insulin resistance and NO has been observed [25]. It has been demonstrated that inhibition of NO syntheses provokes insulin resistance [26, 27], and insulin sensitivity is associated with the release of NO [28, 29]. However, we did not find any association between ADMA, as an endogenous NO inhibitor, and HOMA-IR.

Furthermore, the homocysteine levels did not differ between the GDM and NGT groups in our study. Some researchers found similar homocysteine levels between two groups [30], but in other investigations, a statistically significant difference in homocysteine levels between GDM and NGT group [31, 32] has been observed. Tarim et al. [32] found a relationship between homocysteine and 50-g GCT. In our study, plasma ADMA concentrations were positively correlated with homocysteine only in the NGT group. Homocysteine increases ADMA levels probably by inhibiting the DDAH activity [10, 33, 34], and hyperhomocysteinemia has been found to cause higher ADMA levels [33, 34]. However, in our study, the only factors related to homocysteine levels in multiple regression analysis were serum folic acid and TG levels.

Some previous studies demonstrated a relationship between GDM and inflammatory mediators [35, 36]. We found that hCRP level, which is a marker of inflammation, was significantly higher in the GDM group than the NGT group during late pregnancy. Wolf et al. [36] carried out a prospective study, in which they compared the inflammatory markers including CRP and leukocyte count during first and second trimesters, and found that the first-trimester CRP levels were significantly increased among women who subsequently developed diabetes. Leipold et al. [37] measured the CRP levels during OGTT between the 24th and 28th weeks of gestation, and between the 37th and 38th weeks of gestation, and found that GDM was related to increased CRP level during the third trimester, but not at the time of diagnosis in the second trimester. In their study, the CRP level was correlated with BMI, which is in agreement with our results. According to our results, hCRP was correlated only with the BMI in the whole group and $\mathrm{HbA} 1 \mathrm{c}$ in the GDM group (HbA1c was only analyzed in the GDM group). In addition, we did not find any relationship between ADMA and hCRP levels, which were the atherosclerosis-related markers in our study. This result is similar to a study carried out on healthy pregnant women, in whom a correlation between ADMA and hCRP levels could not be observed in the third trimester [38].

We showed that the GDM group had higher TG levels than the NGT group. In line with our result, Tarim et al. [32] reported increased TG levels in GDM during the second trimester. Another study indicated that TG levels 
Table 3 Spearman correlation analyses between ADMA, hCRP, homocysteine, and the selected variables in GDM group and NGT group

\begin{tabular}{|c|c|c|c|c|c|c|c|c|c|c|c|c|}
\hline & \multicolumn{6}{|c|}{ GDM group } & \multicolumn{6}{|c|}{ NGT group } \\
\hline & \multicolumn{2}{|l|}{ ADMA } & \multicolumn{2}{|c|}{ Homocysteine } & \multicolumn{2}{|l|}{ hCRP } & \multicolumn{2}{|l|}{ ADMA } & \multicolumn{2}{|c|}{ Homocysteine } & \multicolumn{2}{|l|}{ hCRP } \\
\hline & $r$ & $P$ & $r$ & $P$ & $r$ & $P$ & $r$ & $P$ & $r$ & $P$ & $r$ & $P$ \\
\hline ADMA & - & & -0.02 & 0.8 & 0.12 & 0.3 & - & & 0.28 & $\mathbf{0 . 0 3}^{*}$ & -0.02 & 0.8 \\
\hline Homocysteine & -0.02 & 0.8 & - & & 0.13 & 0.3 & 0.28 & $0.03 *$ & - & & -0.008 & 0.9 \\
\hline hCRP & 0.12 & 0.3 & 0.13 & 0.3 & - & & -0.02 & 0.8 & -0.008 & 0.9 & - & \\
\hline Age & 0.25 & 0.6 & 0.24 & 0.08 & 0.10 & 0.4 & -0.06 & 0.5 & 0.17 & 0.1 & -0.19 & 0.1 \\
\hline Gestational week & 0.15 & 0.2 & 0.08 & 0.5 & 0.03 & 0.8 & -0.12 & 0.3 & 0.05 & 0.6 & 0.06 & 0.6 \\
\hline BMI & -0.09 & 0.5 & -0.07 & 0.6 & 0.30 & $0.03 *$ & 0.10 & 0.3 & -0.12 & 0.3 & 0.30 & $0.01 *$ \\
\hline Prepregnancy BMI & 0.08 & 0.5 & -0.09 & 0.5 & 0.16 & 0.2 & 0.12 & 0.3 & -0.02 & 0.8 & 0.15 & 0.2 \\
\hline $50-\mathrm{g}$ GCT & 0.02 & 0.8 & 0.18 & 0.2 & -0.04 & 0.7 & 0.13 & 0.2 & 0.27 & $0.04 *$ & -0.13 & 0.2 \\
\hline FBG & -0.01 & 0.9 & -0.17 & 0.2 & -0.17 & 0.2 & 0.07 & 0.5 & 0.09 & 0.4 & -0.13 & 0.2 \\
\hline HOMA-IR & 0.17 & 0.2 & -0.25 & 0.07 & 0.01 & 0.9 & -0.14 & 0.2 & -0.14 & 0.2 & -0.04 & 0.7 \\
\hline Insulin & 0.19 & 0.1 & -0.19 & 0.1 & 0.10 & 0.4 & -0.15 & 0.2 & -0.16 & 0.2 & -0.03 & 0.7 \\
\hline PPG & -0.20 & 0.1 & -0.05 & 0.7 & -0.08 & 0.5 & -0.04 & 0.7 & 0.12 & 0.3 & 0.07 & 0.5 \\
\hline HbA1c & 0.008 & 0.9 & 0.20 & 0.1 & 0.46 & $0.001 * *$ & - & & - & & - & \\
\hline $\mathrm{TC}$ & 0.07 & 0.5 & 0.26 & 0.06 & -0.10 & 0.4 & -0.04 & 0.7 & 0.19 & 0.1 & 0.09 & 0.4 \\
\hline LDL-C & 0.01 & 0.9 & 0.06 & 0.6 & -0.02 & 0.8 & -0.18 & 0.1 & 0.14 & 0.2 & 0.007 & 0.9 \\
\hline TG & 0.02 & 0.8 & 0.31 & $0.02 *$ & -0.16 & 0.2 & 0.06 & 0.6 & -0.01 & 0.9 & 0.01 & 0.9 \\
\hline HDL-C & 0.20 & 0.1 & 0.08 & 0.5 & 0.06 & 0.6 & 0.16 & 0.1 & 0.06 & 0.6 & 0.08 & 0.5 \\
\hline Folic acid & -0.03 & 0.8 & -0.08 & 0.5 & -0.17 & 0.2 & -0.12 & 0.3 & -0.61 & $<0.001 * *$ & -0.10 & 0.4 \\
\hline B12 & -0.003 & 0.9 & -0.14 & 0.3 & -0.24 & 0.08 & -0.03 & 0.7 & -0.06 & 0.6 & 0.23 & 0.06 \\
\hline
\end{tabular}

GDM gestational diabetes mellitus, $N G T$ normal glucose tolerance, ADMA asymmetric dimethylarginine, $h C R P$ high-sensitive C-reactive protein, $B M I$ body mass index, HOMA-IR homeostasis model assessment for insulin resistance, $F B G$ fasting blood glucose, $P P G$ post-prandial blood glucose, 50-g GCT blood glucose levels after $1 \mathrm{~h} \mathrm{50-g} \mathrm{glucose} \mathrm{challenge} \mathrm{test,} T C$ total cholesterol, $L D L-C$ low-density lipoprotein cholesterol, $H D L-C$ high-density lipoprotein cholesterol, $T G$ triglycerides

$P>0.05$ statistically non-significant, $* P<0.05$, ** $P<0.01$

Bold values indicate statistically significant relationships

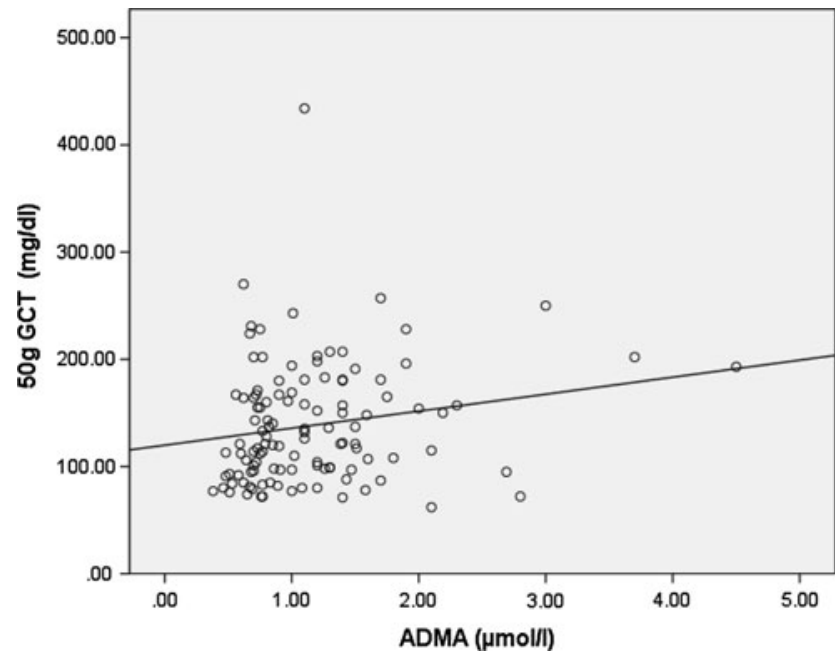

Fig. 2 ADMA concentrations are positively correlated with 1-h glucose at 50-g GCT levels in the whole group

were elevated in pregnancies complicated by diabetes, including GDM [39]. In addition, we found no significant differences in plasma lipids in our previous study, although we found increased levels of small dense LDL in women with GDM during the second trimester [40].

Thus, it can be concluded that plasma ADMA and serum hCRP concentrations are increased in women with GDM during late pregnancy. Our results might imply that endothelial dysfunction and inflammation, which have different mechanisms in atherosclerosis pathogenesis, occur independently in women with GDM during late pregnancy. However, further studies are needed to clarify the significance and the underlying mechanisms of the elevated ADMA and hCRP levels in women with GDM. Furthermore, whether or not the atherosclerosis begins to develop in women with GDM during late pregnancy must be clarified as well.

Acknowledgments We gratefully acknowledge Assoc. Prof. Yasemin Genc and Zeynep Biyıklı for their help in the statistical analysis.

\section{References}

1. B.E. Metzger, T.A. Buchanan, D.R. Coustan, A. de Leiva, D.B. Dunger, D.R. Hadden, M. Hod, J.L. Kitzmiller, S.L. Kjos, J.N. 
Oats, D.J. Pettitt, D.A. Sacks, C. Zoupas, Summary and recommendations of the Fifth International Workshop-Conference on Gestational Diabetes Mellitus. Diabetes Care 30, 251-260 (2007)

2. B.E. Metzger, D.R. Coustan, Summary and recommendations of the Fourth International Workshop-Conference on Gestational Diabetes Mellitus. The Organizing Committee. Diabetes Care 21, 161-167 (1998)

3. E. Anastasiou, J.P. Lekakis, M. Alevizaki, C.M. Papamichael, J. Megas, A. Souvatzoglou, S.F. Stamatelopoulos, Impaired endothelium dependent vasodilatation in women with previous gestational diabetes. Diabetes Care 21, 2111-2115 (1998)

4. M.M. Hannemann, W.G. Liddell, A.C. Shore, P.M. Clark, J.E. Tooke, Vascular function in women with previous gestational diabetes mellitus. J. Vasc. Res. 39, 311-319 (2002)

5. S. Bo, S. Valpreda, G. Menato, C. Bardelli, C. Botto, R. Gambino, C. Rabbia, M. Durazzo, M. Cassader, M. Massobrio, G. Pagano, Should we consider gestational diabetes a vascular risk factor? Atherosclerosis 194, 72-79 (2007)

6. G. Di Cianni, C. Lencioni, L. Volpe, A. Ghio, I. Cuccuru, G. Pellegrini, L. Benzi, R. Miccoli, S. Del Prato, C-reactive protein and metabolic syndrome in women with previous gestational diabetes. Diabetes Metab. Res. Rev. 23, 135-140 (2007)

7. V. Mallika, B. Goswami, M. Rajappa, Atherosclerosis pathophysiology and the role of novel risk factors: a clinicobiochemical perspective. Angiology 58, 513-522 (2007)

8. R.H. Böger, Asymmetric dimethylarginine, an endogenous inhibitor of nitric oxide synthase, explains the "L-arginine paradox" and acts as a novel cardiovascular risk factor. J. Nutr. 134, 2842-2847 (2004)

9. P. Vallance, A. Leone, A. Calver, J. Collier, S. Moncada, Accumulation of an endogenous inhibitor of nitric oxide synthesis in chronic renal failure. Lancet 339, 572-575 (1992)

10. R. Siekmeier, T. Grammer, W. März, Roles of oxidants, nitric oxide, and asymmetric dimethylarginine in endothelial function. J. Cardiovasc. Pharmacol. Ther. 13, 279-297 (2008)

11. V. Bermúdez, F. Bermúdez, G. Acosta, A. Acosta, J. Añez, C. Andara, E. Leal, C. Cano, V. Manuel, R. Hernández, Z. Israili, Molecular mechanisms of endothelial dysfunction: from nitric oxide synthesis to ADMA inhibition. Am. J. Ther. 15, 326-333 (2008)

12. R.H. Böger, R. Maas, F. Schulze, E. Schwedhelm, Asymmetric dimethylarginine (ADMA) as a prospective marker of cardiovascular disease and mortality - an update on patient populations with a wide range of cardiovascular risk. Pharmacol. Res. 60, 481-487 (2009)

13. A.E. Altinova, M. Arslan, A. Sepici-Dincel, M. Akturk, N. Altan, F.B. Toruner, Uncomplicated type 1 diabetes is associated with increased asymmetric dimethylarginine concentrations. J. Clin. Endocrinol. Metab. 92, 1881-1885 (2007)

14. E.P. Wijekoon, M.E. Brosnan, J.T. Brosnan, Homocysteine metabolism in diabetes. Biochem. Soc. Trans. 35, 1175-1179 (2007)

15. M. Wolf, L. Sandler, K. Hsu, K. Vossen-Smirnakis, J.L. Ecker, R. Thadhani, First-trimester C-reactive protein and subsequent gestational diabetes. Diabetes Care 26, 819-824 (2003)

16. C. Zoccali, F.A. Benedetto, R. Maas, F. Mallamaci, G. Tripepi, L.S. Malatino, R. Böger, CREED Investigators, Asymmetric dimethylarginine, C-reactive protein, and carotid intima-media thickness in end-stage renal disease. J. Am. Soc. Nephrol. 13, 490-496 (2002)

17. K. Krzyzanowska, F. Mittermayer, M. Wolzt, G. Schernthaner, Asymmetric dimethylarginine predicts cardiovascular events in patients with type 2 diabetes. Diabetes Care 30, 1834-1839 (2007)

18. F. Mittermayer, B.X. Mayer, A. Meyer, C. Winzer, G. Pacini, O.F. Wagner, M. Wolzt, A. Kautzky-Willer, Circulating concentrations of asymmetrical dimethyl-L-arginine are increased in women with previous gestational diabetes. Diabetologia 45, 1372-1378 (2002)

19. F. Mittermayer, A. Kautzky-Willer, C. Winzer, K. Krzyzanowska, T. Prikoszovich, S. Demehri, O. Wagner, M. Wolzt, Elevated concentrations of asymmetric dimethylarginine are associated with deterioration of glucose tolerance in women with previous gestational diabetes mellitus. J. Intern. Med. 261, 392-398 (2007)

20. B. Telejko, A. Zonenberg, M. Kuzmicki, A. Modzelewska, K. Niedziolko-Bagniuk, A. Ponurkiewicz, A. Nikolajuk, M. Gorska, Circulating asymmetric dimethylarginine, endothelin-1 and cell adhesion molecules in women with gestational diabetes. Acta Diabetol. 46, 303-308 (2009)

21. B.M. Chen, L.W. Xia, R.Q. Zhao, Determination of N(G), N(G)dimethylarginine in human plasma by high-performance liquid chromatography. J. Chromatogr. B 692, 467-471 (1997)

22. G.A. Knock, A.L. McCarthy, C. Lowy, L. Poston, Association of gestational diabetes with abnormal maternal vascular endothelial function. Br. J. Obstet. Gynaecol. 104, 229-234 (1997)

23. K.Y. Lin, A. Ito, T. Asagami, P.S. Tsao, S. Adimoolam, M. Kimoto, H. Tsuji, G.M. Reaven, J.P. Cooke, Impaired nitric oxide synthase pathway in diabetes mellitus: role of asymmetric dimethylarginine and dimethylarginine dimethylaminohydrolase. Circulation 106, 987-992 (2002)

24. A. Biri, A. Onan, E. Devrim, F. Babacan, M. Kavutcu, I. Durak, Oxidant status in maternal and cord plasma and placental tissue in gestational diabetes. Placenta 27, 327-332 (2006)

25. H.O. Steinberg, H. Chaker, R. Leaming, A. Johnson, G. Brechtel, A.D. Baron, Obesity/insulin resistance is associated with endothelial dysfunction. Implications for the syndrome of insulin resistance. J. Clin. Invest. 97, 2601-2610 (1996)

26. A.D. Baron, H.O. Steinberg, H. Chaker, R. Leaming, A. Johnson, G. Brechtel, Insulin-mediated skeletal muscle vasodilation contributes to both insulin sensitivity and responsiveness in lean humans. J. Clin. Invest. 96, 786-792 (1995)

27. A.D. Baron, J.S. Zhu, S. Marshall, O. Irsula, G. Brechtel, C. Keech, Insulin resistance after hypertension induced by the nitric oxide synthesis inhibitor L-NMMA in rats. Am. J. Physiol. 269, 709-715 (1995)

28. K. Sydow, C.E. Mondon, J. Schrader, H. Konishi, J.P. Cooke, Dimethylarginine dimethylaminohydrolase overexpression enhances insulin sensitivity. Arterioscler. Thromb. Vasc. Biol. 28, 692-697 (2008)

29. M. Wohlin, J. Sundstrom, J. Arnlov, B. Andren, B. Zethelius, L. Lind, Impaired insulin sensitivity is an independent predictor of common carotid intima-media thickness in a population sample of elderly men. Atherosclerosis 170, 181-185 (2003)

30. B. Idzior-Waluś, K. Cyganek, K. Sztefko, G. Seghieri, M.C. Breschi, M. Waluś-Miarka, E. Kawalec, M. Seretny, J. Sieradzki, Total plasma homocysteine correlates in women with gestational diabetes. Arch. Gynecol. Obstet. 278, 309-313 (2008)

31. M.A. Guven, M. Kilinc, C. Batukan, H.C. Ekerbicer, T. Aksu, Elevated second trimester serum homocysteine levels in women with gestational diabetes mellitus. Arch. Gynecol. Obstet. 274, 333-337 (2006)

32. E. Tarim, T. Bagis, E. Kilicdag, S. Erkanli, E. Aslan, N. Sezgin, E. Kuscu, Elevated plasma homocysteine levels in gestational diabetes mellitus. Acta Obstet. Gynecol. Scand. 83, 543-547 (2004)

33. R.H. Böger, S.R. Lentz, S.M. Bode-Boger, H.R. Knapp, W.G. Haynes, Elevation of asymmetrical dimethylarginine may mediate endothelial dysfunction during experimental hyperhomocyst(e)inaemia in humans. Clin. Sci. 100, 161-167 (2001)

34. M.C. Stühlinger, P.S. Tsao, J.H. Her, M. Kimoto, R.F. Balint, J.P. Cooke, Homocysteine impairs the nitric oxide synthase pathway. 
Role of asymmetric dimethylarginine. Circulation 104, 2569-2575 (2001)

35. J.P. Kirwan, S. Hauguel-De Mouzon, J. Lepercq, J.C. Challier, L. Huston-Presley, J.E. Friedman, S.C. Kalhan, P.M. Catalano, TNF-a is a predictor of insulin resistance in human pregnancy. Diabetes 51, 2207-2213 (2002)

36. M. Wolf, J. Sauk, A. Shah, K. Vossen Smirnakis, R. JimenezKimble, J.L. Ecker, R. Thadhani, Inflammation and glucose intolerance: a prospective study of gestational diabetes mellitus. Diabetes Care 27, 21-77 (2004)

37. H. Leipold, C. Worda, C.J. Gruber, T. Prikoszovich, O. Wagner, A. Kautzky-Willer, Gestational diabetes mellitus is associated with increased C-reactive protein concentrations in the third but not second trimester. Eur. J. Clin. Invest. 35, 752-757 (2005)
38. P. Valtonen, K. Punnonen, H. Saarelainen, N. Heiskanen, O.T. Raitakari, J.S. Viikari, T. Lyyra-Laitinen, T. Laitinen, S. Heinonen, Maternal serum ADMA is not associated with proinflammatory cytokines or C-reactive protein during normal pregnancy. Cytokine 46, 216-221 (2009)

39. V. Toescu, S.L. Nuttall, U. Martin, P. Nightingale, M.J. Kendall, P. Brydon, F. Dunne, Changes in plasma lipids and markers of oxidative stress in normal pregnancy and pregnancies complicated by diabetes. Clin. Sci. 106, 93-98 (2004)

40. M. Rizzo, K. Berneis, A.E. Altinova, F.B. Toruner, M. Akturk, G. Ayvaz, G.B. Rini, G.A. Spinas, M. Arslan, Atherogenic lipoprotein phenotype and LDL size and subclasses in women with gestational diabetes. Diabet. Med. 25, 1406-1411 (2008) 